\title{
G sonstem \\ Two distinct immunopathological profiles in lungs of lethal COVID-19
}

\section{Ronny Nienhold}

Department of Biomedicine, Experimental Hematology, University Hospital Basel and University of Basel, 4031 Basel, Switzerland

\section{Yari Ciani}

Laboratory of Computational and Functional Oncology, Department for Cellular, Computational and Integrative Biology - CIBIO, University of Trento, Trento, Italy https://orcid.org/0000-0002-9635-2830

\section{Viktor Koelzer}

University of Zurich https://orcid.org/0000-0001-9206-4885

\section{Alexandar Tzankov}

University Hospital Basel https://orcid.org/0000-0002-1100-3819

\section{Jasmin Haslbauer}

Pathology, Institute of Medical Genetics and Pathology, University Hospital Basel, Basel, Switzerland

\section{Thomas Menter}

Pathology, Institute of Medical Genetics and Pathology, University Hospital Basel, Basel, Switzerland

\section{Nathalie Schwab}

Institute of Pathology, Cantonal Hospital Baselland, Liestal, Switzerland

\section{Maurice Henkel}

Institute of Pathology, Cantonal Hospital Baselland, Liestal, Switzerland https://orcid.org/0000-0002-

7560-0938

\section{Angela Frank}

Institute of Pathology, Cantonal Hospital Baselland

\section{Veronika Zsikla}

Institute of Pathology, Cantonal Hospital Baselland, Liestal, Switzerland

\section{Niels Willi}

Institute of Pathology, Cantonal Hospital Baselland, Liestal, Switzerland

\section{Werner Kempf}

Kempf und Pfaltz Histologische Diagnostik, Zurich, Switzerland

\section{Thomas Hoyler}

Novartis Institutes for BioMedical Research (NIBR), Basel, Switzerland

\section{Mattia Barbareschi}

U.O. Anatomia Patologica Ospedale S. Chiara https://orcid.org/0000-0003-0217-3223

\section{Holger Moch}

University Hospital of Zurich 


\section{Markus Tolnay}

University Hospital Basel

\section{Gieri Cathomas}

Institute of Pathology, Cantonal Hospital Baselland, Liestal, Switzerland

\section{Francesca Demichelis}

University of Trento https://orcid.org/0000-0002-8266-8631

\section{Tobias Junt}

Novartis Pharma

\section{Kirsten Mertz ( $\square$ kirsten.mertz@ksbl.ch )}

Institute of Pathology, Cantonal Hospital Baselland, Liestal, Switzerland

\section{Article}

Keywords: COVID-19, immune responses, treatment

Posted Date: July 24th, 2020

DOI: https://doi.org/10.21203/rs.3.rs-37729/v1

License: (c) (i) This work is licensed under a Creative Commons Attribution 4.0 International License. Read Full License

Version of Record: A version of this preprint was published at Nature Communications on October 8th, 2020. See the published version at https://doi.org/10.1038/s41467-020-18854-2. 


\section{Abstract}

Immune responses in lungs of Coronavirus Disease 2019 (COVID-19) are poorly characterized. We conducted transcriptomic, histologic and cellular profiling of post mortem COVID-19 and normal lung tissues. Two distinct immunopathological reaction patterns were identified. One pattern showed high expression of interferon stimulated genes (ISGs) and cytokines, high viral loads and limited pulmonary damage, the other pattern showed severely damaged lungs, low ISGs, low viral loads and abundant immune infiltrates. Distinct patterns of pulmonary COVID-19 immune responses correlated to hospitalization time and may guide treatment and vaccination approaches.

\section{Main Text}

COVID-19 is a pandemic respiratory disease with $5-10 \%$ lethality ${ }^{1}$. While a coronavirus (SARS-CoV-2) is the causative agent, the antiviral immune response may also contribute to devastating end organ damage ${ }^{2}$. Therefore analysis of post mortem tissues is critical for the mechanistic understanding of COVID-19 pathophysiology ${ }^{3}$.

Here we analyzed 34 post mortem lung samples from 16 deceased COVID-19 patients and 9 post mortem lung samples from 6 patients who died from non-infectious causes (Extended Data Table 1). Of note, we did not find bacterial superinfections in COVID-19 lungs except in three samples (Extended Data Figure 1). Differential gene expression analysis based on a commercial assay (OIRRA) designed for quantification of immune cell and inflammatory transcripts (Extended Data Table 2) identified 68 upregulated and 30 down-regulated genes in COVID-19 infected lungs compared to normal tissue (Extended Data Table 3). Using a consensus of 30 different indices ${ }^{4}$ we identified by K-means clustering three groups of samples defined by the expression levels of the deregulated genes (Figure 1a, Extended Data Figure 2). Clusters 1 (50\% of samples) and 2 (41\%) contained COVID-19 samples and cluster 3 contained all normal lung samples and 3 COVID-19 samples (9\%). To understand why the majority of COVID-19 lung tissues segregated into defined clusters 1 or 2 , we undertook a gene ontology analysis. We identified interferon stimulated genes (ISGs) as a key upregulated pathway in COVID-19 autopsy lungs (Figure 1b), which was differentially represented in cluster 1 and 2, respectively (Figure 1c). Identification of an ISG high cluster (Cluster 1, ISG ${ }^{\text {high }}$ ) was surprising, since SARS-CoV-2 was proposed to lead to limited ISG induction, yet only in comparison to other respiratory viruses ${ }^{5}$. Next, we analyzed frequencies of specific immune cells in lungs by computational image analysis. T cells (CD3+), particularly CD8+ T cells, and macrophages (CD68+, CD163+) were selectively enriched in samples from the ISG ${ }^{\text {low }}$ cluster 2 (Figure 1d), as were CD4+ T cells, while CD20+ B cells and CD123+ plasmacytoid dendritic cells (pDCs) did not show differential frequencies between ISG ${ }^{\text {high }}$ and ISG ${ }^{\text {low }}$ lungs (Extended Data Figure $3 a, b$ ). Since T cell exhaustion was observed in COVID-196, we compared frequencies of CD8+PD1+ cells in ISG ${ }^{\text {high }}$ and ISG ${ }^{\text {low }}$ samples, and observed a higher frequency of CD8+PD1+ T-cells in the ISG ${ }^{\text {low }}$ subgroup, potentially indicative of advanced disease (Extended Data Figure 3a,b). Histological analysis of COVID-19 lung 
tissues revealed striking pulmonary damage exclusively in ISG ${ }^{\text {low }}$ samples, with distinct peri-alveolar foci of infiltrating CD68+ macrophages and CD8+ T cells (Figure 1e).

Expression of ISGs was tightly correlated with pulmonary viral load (Figure 2a), and immunohistochemical staining showed SARS-CoV-2 nucleocapsid protein mostly in pneumocytes of ISG ${ }^{\text {high }}$ lungs (Figure $2 \mathrm{~b}$ ). Since a cytokine storm has been proposed to cause adverse outcome of COVID$19^{7}$, and cytokines were highly expressed in bronchoalveolar lavages (BALs) of COVID-19 patients ${ }^{8}$, we investigated expression of a pro-inflammatory cytokine signature (TNF, IL6, IL1b, CCL2, IFNA17, IFNB1, CXCL9, CXCL10, CXCL11) in lung samples from lethal COVID-19. The proinflammatory gene signature was significantly enriched in the ISG ${ }^{\text {high }}$ subset (Figure 2c), but was not associated with alveolar hemorrhage (Figure 2d). Within this cytokine signature, co-regulated subgroups (IL1B/IL6/TNF, IFNB1/IFNA17, CCL2/CXCL9/CXCL19/CXCL11) were identified (Figure 2e). Importantly, only the CXCL9/10/11 sub-signature was positively associated with alveolar hemorrhage (Figure 2f, Extended Data Figure 4). This is in line with observations that these chemokines compromise endothelial integrity via $\mathrm{CXCR}^{9}$, and that $\mathrm{CXCL} 10$ is a key determinant of severe COVID-19 ${ }^{10}$. Interestingly, basal levels of CXCL9 or CXCL10 are elevated in elderly, hypertensive and obese individuals, which were strongly represented in our autopsy cohort and are predisposed to severe COVID-1911,12. Of note, our study could not take extrapulmonary cytokine sources or effects into account.

Since none of the above pulmonary cytokine sub-signatures was positively associated with diffuse alveolar damage (DAD, Extended Data Figure 5), we investigated which other local immune signature showed this association. We found a strong association of DAD with low expression of ISGs (Figure $\mathbf{2 g}$ ), and an activated CD8+ $T$ cell signature (CD38, GZMA, GZMB, CCR5, Figure $2 h$ ), yet not with pulmonary CD8+ T-cell infiltration (Figure 2i). In addition, the activated CD8+ signature was inversely correlated to viral counts, particularly in ISG ${ }^{\text {low }}$ cases (Figure 2j). Therefore we speculate that activated CD8+ T cells are essential for virus elimination, similar to murine models of coronavirus infection ${ }^{13}$, yet it is possible that they contribute to pulmonary damage as well. Of note, ISG ${ }^{\text {low }}$ samples also expressed elevated p53 and Ki67 (Figure 2k), i.e. reactive markers of DAD which indicate lung remodeling ${ }^{14}$. Since the ISG $\mathrm{G}^{\text {low }}$ pattern showed lower viral counts, higher accumulation and activation of CD8+ T cells in tissues and accrual of pulmonary damage and remodeling, the ISG ${ }^{\text {low }}$ phase may follow an earlier ISG ${ }^{\text {high }}$ phase during the course of infection. This was supported by significantly longer hospitalization in COVID-19 patients from whom ISG low lung samples were obtained (Figure 2l). This is in line with epidemiologic data showing that one defined group of critical COVID-19 patients quickly succumbs to disease after hospitalization, while others die after longer intensive care $^{15}$.

Discovery of two patterns of immunopathology in COVID19 post mortem lungs suggests that only patients with an ISG ${ }^{\text {high }}$ pattern show high viral loads and may benefit from antivirals. ISG ${ }^{\text {low }}$ patients instead show low viral loads yet strong complement activation in lungs (Figure $2 \mathrm{~m}$ ) and thus may potentially benefit from complement inhibition ${ }^{16}$. In addition, the ISG ${ }^{\text {low }}$ pattern suggests that CD $8+T$ cells are involved in antiviral protection and should be considered for vaccination efforts. 


\section{Methods}

\section{Ethics statement}

This study was conducted according to the principles expressed in the Declaration of Helsinki. Ethics approval was obtained from the Ethics Committee of Northwestern and Central Switzerland (Project-ID 2020-00629). For all patients, either personal and/or family consent was obtained for autopsy and sample collection.

\section{Patients and sample collection}

The study is based on the analysis of 16 out of 21 consecutive COVID-19 autopsies performed between March $9^{\text {th }}$ and April $14^{\text {th }} 2020$ at the Institute of Pathology Liestal and Institute of Medical Genetics and Pathology Basel, Switzerland. Clinical features including symptoms, course of disease, comorbidities, laboratory results and therapy are listed in Extended Data Table 1a. Detailed autopsy findings for each patient were recently published, and the identifiers (with the prefix " $C^{\prime \prime}$ ) for each COVID-19 patient are consistent with the description of this Swiss COVID-19 autopsy cohort ${ }^{3}$. In this study, we analysed formalin fixed and paraffin embedded (FFPE) lung tissue of distinct areas of the lungs of 16 of these COVID-19 patients. All 16 COVID-19 patients had positive nasopharyngeal swabs collected while alive. In all COVID-19 patients, diagnosis was confirmed by detection of SARS-CoV-2 in postmortal lung tissues. $5 / 16$ patients were additionally tested by postmortal nasopharyngeal swabs which were positive for SARS-CoV-2 in all 5 cases.

As a control cohort, we selected 6 autopsies performed between January 2019 and March 2020 at the Institute of Pathology Liestal ("normal" patients N1 - N6). These control patients died of other, noninfectious causes and had a similar age, gender and cardiovascular risk profile. Patients with infections were excluded from this control cohort. Another control cohort consisted of 4 autopsies of patients suffering from various infections mainly with bacteria affecting the lung (patients with lung pathology, P1 - P4). Details for both control cohorts are listed in Extended Data Table 1b,c. SARS-CoV-2 was ruled out for each control patient by PCR-examination of lung tissue samples.

\section{Nucleic acid extraction}

RNA was extracted from up to six sections of FFPE tissue blocks using RecoverAll Total Nucleic Acid Isolation Kit (Cat No. AM1975, Thermo Fisher Scientific, Waltham, MA, USA). Extraction of DNA from up to 10 sections of FFPE tissue samples was automated by EZ1 Advanced XL (Qiagen, Hilden, Germany) using the EZ1 DNA Tissue Kit (Cat No. 953034, Qiagen, Hilden, Germany). Concentration of DNA and RNA were measured with Qubit 2.0 Fluorometer and Qubit dsDNA HS Assay or Qubit RNA HS Assay (Cat No. Q33230 \& Q32852, Thermo Fisher Scientific, Waltham, MA, USA), respectively.

\section{Quantification of SARS-CoV-2 in FFPE tissue samples}


Post mortem viral load was individually measured in all lung tissue blocks from all patients included in this study. SARS-CoV-2 was detected in 15ng of human total RNA using the TaqMan 2019-nCoV Assay Kit v1 (Cat No. A47532, Thermo Fisher Scientific, Waltham, MA, USA), which targets three genomic regions (ORFab1, S Protein, N Protein) specific for SARS-CoV-2 and the human RNase P gene (RPPH1). The copy numbers of the SARS-CoV-2 viral genome was determined by utilizing the TaqMan 2019-nCoV Control Kit v1 (Cat No. A47533, Thermo Fisher Scientific, Waltham, MA, USA) and a comparative " $\triangle \Delta C \mathrm{CT}^{\prime}$ method. The control kit contains a synthetic sample with a defined amount of target molecules for the human RPPH1 and the three SARS-CoV-2 assays, and was re-analyzed in parallel with patient samples. For each patient sample, this method resulted in individual copy numbers of the human RPPH1 and the three SARS-CoV-2 targets. Finally, the mean copy number of the SARS-CoV-2 targets was normalized to 1 $x 10^{6}$ RPPH 1 transcripts.

\section{Profiling of immune response by targeted RNAseq}

The expression levels of 398 genes, including genes relevant in innate and adaptive immune response and housekeeping genes for normalization, were analyzed with the Oncomine Immune Response Research Assay (OIRRA, Cat No. A32881, Thermo Fisher Scientific, Waltham, MA, USA). The OIRRA is a targeted gene expression assay designed for the lon $^{\text {TM }}$ next-generation sequencing (NGS) platform. This gene expression assay was originally designed to interrogate the tumor microenvironment to enable mechanistic studies and identification of predictive biomarkers for immunotherapy in cancer. The assay is optimized to measure the expression of genes involved in immune cell interactions and signaling, including genes expressed at low levels and involved in inflammatory signaling. The 398 genes covered by this assay are listed in Extended Data Table 2.

The NGS libraries were prepared as recommended by the supplier. In brief, 30ng of total RNA were used for reverse transcription (SuperScript VILO, Cat No. 11754250, Thermo Fisher Scientific, Waltham, MA, USA) and subsequent library preparation. The libraries were quantified (Ion Library TaqMan Quantitation Kit, Cat No. 4468802, Thermo Fisher Scientific, Waltham, MA, USA), equimolarly pooled and sequenced utilizing the lon GeneStudio S5xI (Thermo Fisher Scientific, Waltham, MA, USA). De-multiplexing and gene expression level quantification were performed with the standard setting of the ImmuneResponseRNA plugin (version 5.12.0.1) within the Torrent Suite (version 5.12.1), provided as part of the OIRRA by Thermo Fisher Scientific, Waltham, MA, USA.

\section{Detection of co-infections by whole genome sequencing}

To identify potential pathogens accompanying an infection with SARS-CoV-2, we analyzed the DNA of the same tissue samples used for detection and profiling of the SARS-CoV-2-specific immune response. First, $250 \mathrm{ng}$ of genomic DNA was enzymatically sheared $\left(15\right.$ minutes at $\left.37^{\circ} \mathrm{C}\right)$ and barcoded using the Ion Xpress Plus Fragment Library Kit (Cat No. 4471269, Thermo Fisher Scientific, Waltham, MA, USA). Subsequently, the libraries were quantified (Ion Library TaqMan Quantitation Kit, Cat No. 4468802, Thermo Fisher Scientific, Waltham, MA, USA) and up to three libraries were pooled at equimolar levels for 
analysis with Ion GeneStudio S5xI (Thermo Fisher Scientific, Waltham, MA, USA). Sequencing data for each sample was analysed using the CLC genomics workbench (version 20.0.3, Qiagen, Hilden, Germany) in combination with the microbial genomics module (version 20.0.1, Qiagen, Hilden, Germany): The raw reads were trimmed by quality (Mott algorithm with limit 0.05 and a maximum of 2 ambiguous bases per read) and mapped to the human genome (GRCh37 hg19, match score: 1, mismatch cost: 2 , indel opening cost: 6 , indel extension cost: 1 ). Unmapped reads were analysed by taxonomic profiling to identify reads of viral or bacterial origin. The profiling utilized an index of 11'540 viral genomes with a minimum length of 1'000 bp and 2'715 bacterial reference genomes with a minimum length of 500 '000 bp, retrieved from the NCBI Reference Sequence Database (date of download: 2020-04-02).

\section{Immunohistochemistry}

Immunohistochemical analyses for CD3, CD4, CD8, CD15, CD20, CD68, CD123, CD163, PD-1, MP0, p53, Ki67, C3d and C5b-9 were performed on all lung tissue blocks used in this study. Antibodies, staining protocols and conditions are detailed in Extended Data Table 4.

\section{Qualitative and semiquantitative assessment of histopathological lung damage and neutrophilic infiltration}

Hematoxylin and eosin (H\&E) and Elastica van Gieson (EvG) stained sections of all lung tissues used in this study were independently evaluated by two experienced and board certified pathologists (VZ and KDM) (Extended Data Table 5). Both pathologists evaluated the presence of diffuse alveolar damage (DAD), and if present, its stage, intra-alveolar edema and hemorrhage. In addition, both pathologists evaluated the severity of histopathological changes in COVID-19 lungs $(1=$ mild $/$ discrete alterations, $2=$ moderate, 3 = severe changes) based on resemblance between normal and pathologically altered lung tissues. Parameters that were taken into account included reduction of alveolar air-filled spaces, typical histologic features of DAD with hyaline membrane formation, infiltration of lymphocytes, monocytes and neutrophils into interstitial and alveolar spaces, type 2 pneumocyte hyperplasia, desquamation of pneumocytes, histologic features of organizing pneumonia including intra-alveolar fibrin deposition and fibrosis (acute fibrinous and organizing pneumonia, AFOP) ${ }^{17,18}$. The number of neutrophils per lung tissue section was estimated on H\&E stained sections and by immunohistochemical stains for CD15 and MPO using a three tiered system ( $1=$ few or no neutrophils, $2=$ moderate number of neutrophils, $3=$ high number of neutrophils). Assessment of the two pathologists was concordant in the vast majority of cases. Discrepant cases were reviewed by a third pathologist (NW) to reach consent.

\section{Digital image analysis}

Slides were digitalized on a 3DHistech ${ }^{\mathrm{TM}} \mathrm{P} 1000$ slide scanner at 400x magnification (3DHISTECH Ltd. Budapest, Hungary). Digital slide review and quality control was performed by a board-certified pathologist (VHK). Tissue regions with staining artefacts, folds or other technical artefacts were excluded from analysis. A deep neural network (DNN) algorithm (Simoyan and Zisserman VGG, HALO A ${ }^{\mathrm{TM}}$ on HALO $^{\text {TM }}$ 3.0.311.167, Indica Labs, Corrales, NM) was trained using pathologist annotations to 
automatically localize and measure the area of each lung tissue sample on the digital slides. Background regions and glass were excluded from analysis. Mark-up images for tissue classification were generated and classification accuracy was confirmed through pathology review. For cell-level analysis, color deconvolution for $\mathrm{DAB}, \mathrm{AP}$ and hematoxylin channels was performed and nuclear segmentation was optimized using cell-morphometric parameters. Marker-positive cells in stromal and epithelial regions were quantified. For CD3, CD4, CD8, CD20, CD68, CD123, CD163 and PD1, staining detection was optimized for the cytoplasmic / membranous compartment and marker expression was measured on a continuous scale at single cell resolution. For assessment of CD8/PD1 double stains, color deconvolution was optimized for separation of DAB (PD1) and AP (CD8) staining products. Internal controls (nonimmune cells) and external controls (tonsil) were used to calibrate the detection limits and crossvalidated by visual review. For each tissue sample, the total area of lung tissue in $\mathrm{mm}^{2}$, the absolute number of marker-positive cells, cell morphometric parameters and staining intensity were recorded.

\section{Identification of SARS-CoV-2 immune response pattern}

\section{Gene expression analysis}

Samples were included in the study based on quality of libraries and alignment performance. Applied inclusion criteria are: $>1$ million of mapped reads, good concentration of libraries, average read length $>100 \mathrm{bp},>300$ target genes with more than 10 reads. One sample with $>1$ Mio reads was excluded from the study because of shorter read length and a low library concentration. Notably this sample had the longest time between death and autopsy (72h) before analysis. Differential expression analysis was performed using the edgeR package comparing normal lung samples, COVID-19 samples and samples from patients with other infections. Genes were selected for downstream analyses by $\mathrm{fdr}<0.05$ and $|\log \mathrm{FC}|>1$ for clustering analysis. Clustering analysis was performed using k-means algorithm and complete linkage. Ideal number of clusters $(n=3)$ was chosen based on 30 different algorithms ${ }^{19}$ and the final clustering derives from the consensus of 2000 iterations. Expression of gene signatures was calculated as median of $\log 2(\mathrm{cpm}+1)$ of selected genes.

\section{Functional enrichment analysis}

Biological processes enrichment was performed using the enrichGO function of the package clusterProfiler ${ }^{20}$ setting all the genes included in the assay as universe.

\section{Statistical analysis}

All the analyses and graphical representations were performed using the $\mathrm{R}$ statistical environment software ${ }^{21}$ and the following packages: ggplot $2^{22}$, circlize $^{23}$, ComplexHeatmap ${ }^{24}$, ggfortify ${ }^{25}$, reshape ${ }^{26}$ and factoextra ${ }^{27}$. Correlation between transcripts and viral counts was performed using Pearson's correlation. Association between continuous and categorical data were tested using Wilcoxon rank sum test. 
Box-plots elements indicate the median (center line), upper and lower quartiles (box limits) and show all the data points. Whiskers extend to the most extreme value included in $1.5 x$ interquartile range.

\section{Declarations}

\section{Data availability}

The datasets generated and analysed during this study can be accessed in GEO (accession number pending) and are available from the corresponding author upon request.

\section{Acknowledgements}

VHK gratefully acknowledges funding by the Promedica Foundation (F-87701-41-01). AT, JDH, TM and KDM are supported by and gratefully acknowledge the Botnar Research Centre for Child Health. We would like to thank Angela Frank, Christian Tosch, Beat Béni, Daniel Turek, Melanie Sachs, Anne Graber, Christina Herz, Arbeneshe Berisha, Norbert Wey and the USZ pathology IT team, André Fitsche, Marcel Glönkler, Christiane Mittman and the USZ pathology laboratory team for expert technical support and scanning of slides.

\section{Author contributions}

RN, YC, VHK, FD, TJ and KDM jointly conceived the study, performed data interpretation and prepared the manuscript. $A T, M B, H M, M T$ and $C A$ provided intellectual input, provided critical resources and critically reviewed the manuscript. $\mathrm{RN}, \mathrm{YC}, \mathrm{VHK}, \mathrm{MH}, \mathrm{TH}$ and $\mathrm{TJ}$ performed bioinformatic and statistical analysis. AT, JDH, TM, NS collected autopsy specimens and patient data. VZ, NW, WK and CA performed histomorphological evaluation. All authors approved the final manuscript.

\section{Competing interests}

VHK has served as an invited speaker on behalf of Indica Labs. TH and TJ are employees of Novartis. The other authors declare no competing interests.

\section{References}

1. Hopkins, J. Corona virus resource center. Latest update: 05/22/2020: https://coronavirus. jhu. edu/data (2020).

2. Chen, G., et al. Clinical and immunological features of severe and moderate coronavirus disease 2019. The Journal of clinical investigation 130(2020).

3. Menter, T., et al. Post-mortem examination of COVID19 patients reveals diffuse alveolar damage with severe capillary congestion and variegated findings of lungs and other organs suggesting vascular dysfunction. Histopathology (2020). 
4. Charrad, M., Ghazzali, N., Boiteau, V. \& Niknafs, A. NbClust Package: finding the relevant number of clusters in a dataset. J. Stat. Softw (2012).

5. Blanco-Melo, D., et al. Imbalanced host response to SARS-CoV-2 drives development of COVID-19. Cell (2020).

6. Diao, B., et al. Reduction and functional exhaustion of T cells in patients with coronavirus disease 2019 (COVID-19). Frontiers in Immunology 11, 827 (2020).

7. Huang, C., et al. Clinical features of patients infected with 2019 novel coronavirus in Wuhan, China. The lancet 395, 497-506 (2020).

8. Liao, M., et al. Single-cell landscape of bronchoalveolar immune cells in patients with COVID-19. Nature Medicine, 1-3 (2020).

9. Bodnar, R.J., Yates, C.C., Rodgers, M.E., Du, X. \& Wells, A. IP-10 induces dissociation of newly formed blood vessels. Journal of cell science 122, 2064-2077 (2009).

10. Yang, Y., et al. Exuberant elevation of IP-10. MCP-3 and IL-1ra during SARS-CoV-2 infection is associated with disease severity and fatal outcome. medRxiv 2002, 2020 (2020).

11. Hueso, L., et al. Upregulation of angiostatic chemokines IP-10/CXCL10 and I-TAC/CXCL11 in human obesity and their implication for adipose tissue angiogenesis. Int J Obes (Lond) 42, 1406-1417 (2018).

12. Bonfante, H.L., et al. CCL2, CXCL8, CXCL9 and CXCL10 serum levels increase with age but are not altered by treatment with hydroxychloroquine in patients with osteoarthritis of the knees. Int $\mathrm{J}$ Rheum Dis 20, 1958-1964 (2017).

13. Cupovic, J., et al. Central Nervous System Stromal Cells Control Local CD8(+) T Cell Responses during Virus-Induced Neuroinflammation. Immunity 44, 622-633 (2016).

14. Shetty, S.K., et al. p53 and miR-34a Feedback Promotes Lung Epithelial Injury and Pulmonary Fibrosis. Am J Pathol 187, 1016-1034 (2017).

15. Salje, H., et al. Estimating the burden of SARS-CoV-2 in France. Science (2020).

16. Magro, C., et al. Complement associated microvascular injury and thrombosis in the pathogenesis of severe COVID-19 infection: a report of five cases. Transl Res (2020).

17. Copin, M.-C., Parmentier, E., Duburcq, T., Poissy, J. \& Mathieu, D. Time to consider histologic pattern of lung injury to treat critically ill patients with COVID-19 infection. Intensive Care Medicine, 1-3 (2020).

18. Xu, Z., et al. Pathological findings of COVID-19 associated with acute respiratory distress syndrome. Lancet Respir Med 8, 420-422 (2020).

19. Bates, D., Mächler, M., Bolker, B. \& Walker, S. Fitting linear mixed-effects models using Ime4. arXiv preprint arXiv:1406.5823 (2014).

20. Chikina, M., Robinson, J.D. \& Clark, N.L. Hundreds of genes experienced convergent shifts in selective pressure in marine mammals. Molecular biology and evolution 33, 2182-2192 (2016).

21. Team, R.C. R: A language and environment for statistical computing. (2013). 
22. Wickham, H. ggplot2: elegant graphics for data analysis, (Springer, 2016).

23. Gu, Z., Gu, L., Eils, R., Schlesner, M. \& Brors, B. circlize implements and enhances circular visualization in R. Bioinformatics 30, 2811-2812 (2014).

24. Gu, Z., Eils, R. \& Schlesner, M. Complex heatmaps reveal patterns and correlations in multidimensional genomic data. Bioinformatics 32, 2847-2849 (2016).

25. Horikoshi, M. \& Tang, Y. ggfortify: Data visualization tools for statistical analysis results. v0. 1.0. URL http://CRAN. R-project. org/package= ggfortify. R package version 0.4 1, 28 (2018).

26. Wickham, H. Reshaping data with the reshape package. Journal of statistical software $21,1-20$ (2007).

27. Kassambara, A. \& Mundt, F. Package 'factoextra'. Extract and visualize the results of multivariate data analyses 76(2017).

\section{Figures}


a

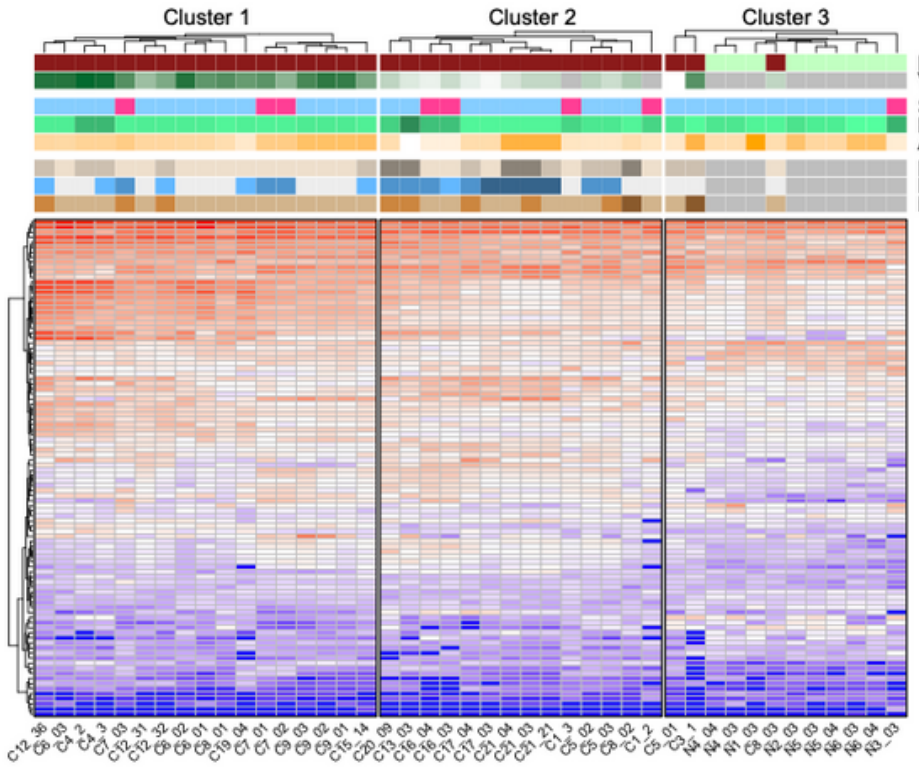

Figure 1

Piagnosis

Sex
AMI
Age

Histo stage

Neutrophils

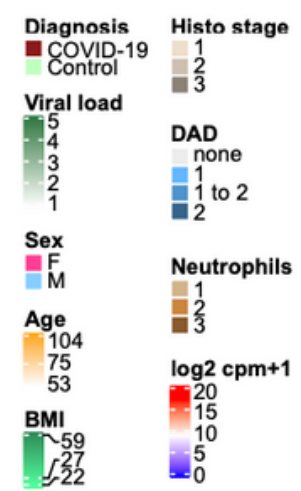

b

\begin{tabular}{|l|c|c|c|c|c|}
\hline ID & Description & GeneRatio & BgRatio & pvalue & p.adjust \\
\hline GO:0034340 & response to type linterferon & $17 / 66$ & $30 / 379$ & $3.70 \mathrm{E}-07$ & 0.000158 \\
\hline GO:0060337 & type linterferon signaling pathway & $17 / 66$ & $30 / 379$ & $3.70 \mathrm{E}-07$ & 0.000158 \\
\hline GO:0071357 & cellular response to type linterferon & $17 / 66$ & $30 / 379$ & $3.70 \mathrm{E}-07$ & 0.000158 \\
\hline GO:0051607 & defense response to virus & $21 / 66$ & $44 / 379$ & $5.53 \mathrm{E}-07$ & 0.000177 \\
\hline GO:0098542 & defense response to other organism & $25 / 66$ & $71 / 379$ & $3.85 \mathrm{E}-05$ & 0.009888 \\
\hline GO:0009615 & response to virus & $21 / 66$ & $57 / 379$ & $9.79 \mathrm{E}-05$ & 0.020960 \\
\hline GO:0045069 & regulation of viral genome replication & $10 / 66$ & $18 / 379$ & $1.87 \mathrm{E}-04$ & 0.034381 \\
\hline GO:1903900 & regulation of viral life cycle & $11 / 66$ & $22 / 379$ & $2.95 \mathrm{E}-04$ & 0.042931 \\
\hline GO:0045071 & negative regulation of viral genome replication & $8 / 66$ & $13 / 379$ & $3.57 \mathrm{E}-04$ & 0.042931 \\
\hline GO:1903901 & negative regulation of viral life cycle & $8 / 66$ & $13 / 379$ & $3.57 \mathrm{E}-04$ & 0.042931 \\
\hline GO:0048525 & negative regulation of viral process & $9 / 66$ & $16 / 379$ & $3.68 \mathrm{E}-04$ & 0.042931 \\
\hline GO:0051603 proteolysis involved in cellular protein catabolic process & $7 / 66$ & $11 / 379$ & $6.71 \mathrm{E}-04$ & 0.071789 \\
\hline GO:0050792 & regulation of viral process & $12 / 66$ & $28 / 379$ & $9.17 \mathrm{E}-04$ & 0.088399 \\
\hline GO:0019079 & viral genome replication & $10 / 66$ & $21 / 379$ & $9.64 \mathrm{E}-04$ & 0.088399 \\
\hline GO:0043901 & negative regulation of multi-organism process & $9 / 66$ & $18 / 379$ & $1.16 \mathrm{E}-03$ & 0.099123 \\
\hline
\end{tabular}

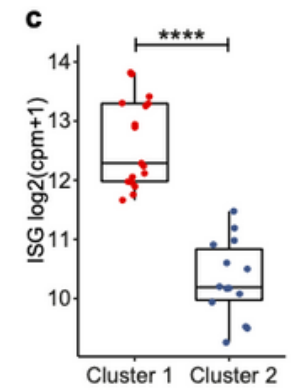

d
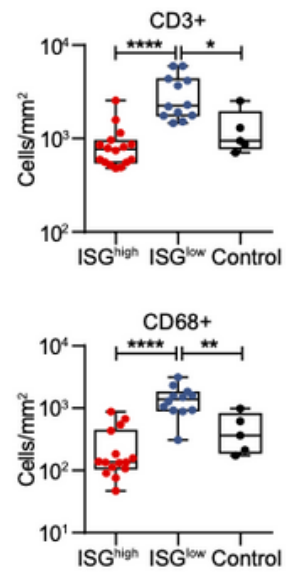

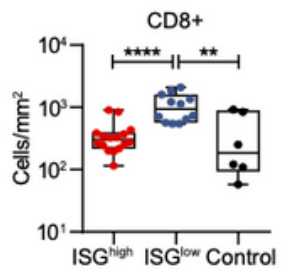

CD163+

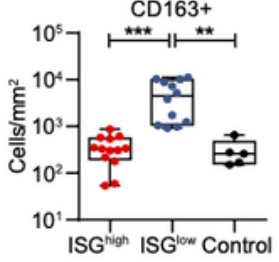

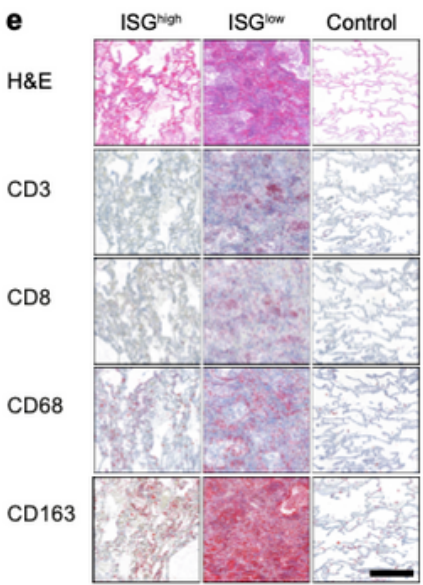

\section{Figure 1}

Transcriptomic and immunologic profile of COVID-19 autopsy lungs. (a) Heatmap showing K-means clustering of COVID-19 and normal lung samples based on expression levels of deregulated genes in COVID-19 versus normal lungs. (b) Gene ontology enrichment analysis of genes upregulated in COVID-19 samples. (c) ISG signature expression in clusters 1 and 2 of COVID-19 lungs. (d) Frequencies of immune cells on ISGhigh and ISGlow COVID-19 lung sections and controls. (e) Representative H\&E stains and 
immunohistochemistry (CD3, CD8, CD68, CD163) of ISGhigh and ISGlow COVID-19 lungs and controls, size bar $500 \mu \mathrm{m}$

Figure 2
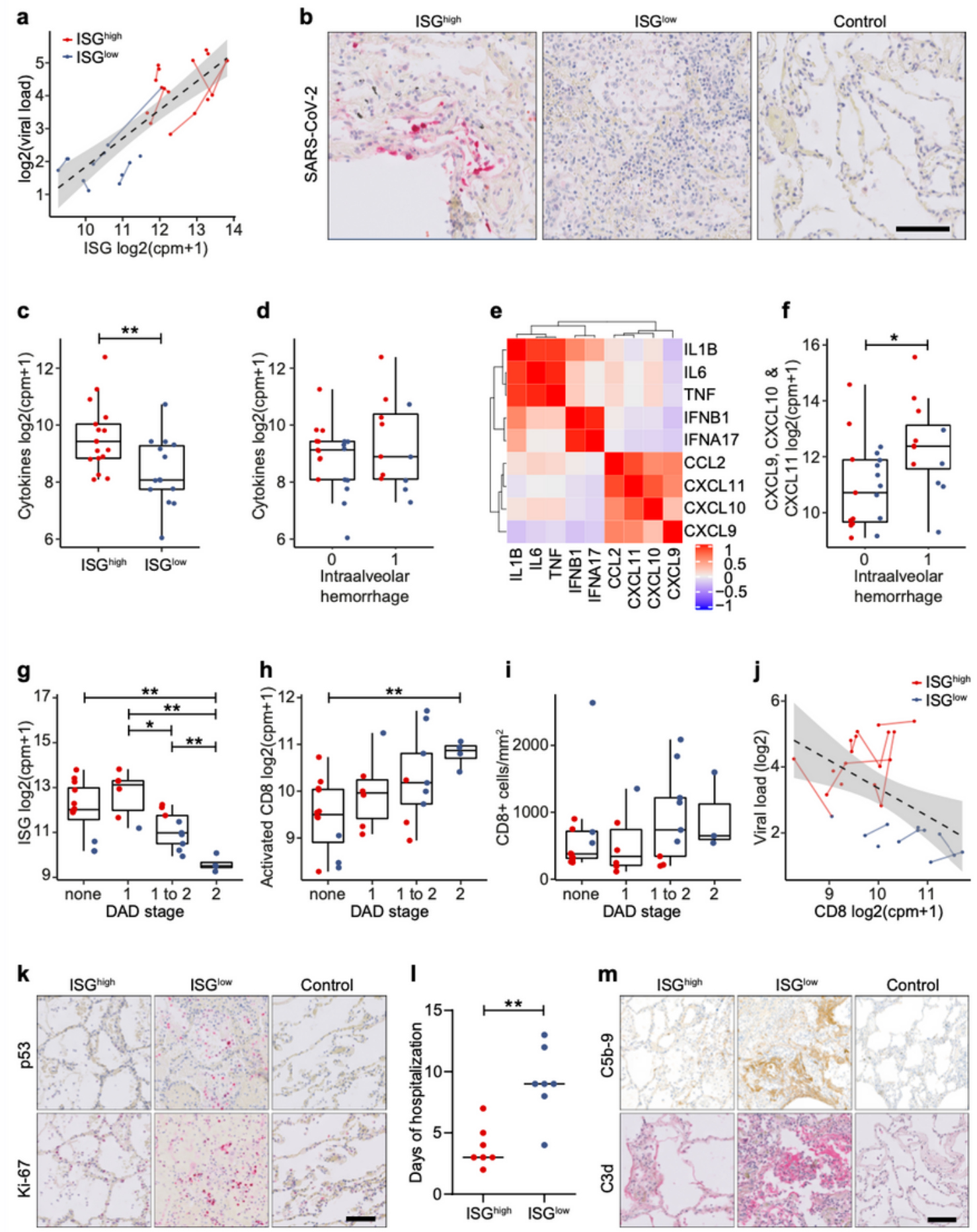

\section{Figure 2}

Relationship between cytokine signature and histopathological changes. (a) Correlation of viral load and ISG expression in COVID-19 lungs. Solid lines, sample data from the same patient. Dotted line, regression for all samples and 95\% Cl (Pearson's correlation=0.83, adjusted R-squared=0.68, p-value=1.66e-08). (b) 
Representative immunohistochemistry for SARS-CoV-2 on ISGhigh and ISGlow COVID-19 lung samples and controls. Size bar $100 \mu \mathrm{m}$. (c) Expression of a cytokine signature (IL-1b, IL6, TNF, IFNa17, IFNb1, CCL2, CXCL9, CXCL10, CXCL11) in ISGhigh and ISGlow COVID-19 lung samples. (d) Association of the cytokine signature with intraalveolar hemorrhage. (e) Pearson's correlation of pro-inflammatory cytokines in the cytokine signature indicates presence of co-regulated cytokines. (f) Association of CXCL9/10/11 expression in lung samples with intraalveolar hemorrhage. Association of DAD stage with (g) ISG expression, (h) activated CD8+ T cell signature, (i) CD8 T cell counts. (j) Inverse correlation of viral load and activated CD8+ $T$ cell signature. Solid line, sample data from the same patient. Dotted line, regression for all the samples and $95 \% \mathrm{Cl}$ (Pearson's correlation=- 0.5 adjusted R-squared $=0.22, \mathrm{p}$ value $=0.005$ ). (k) Representative immunohistochemistry for p53 and Ki67. Size bar $100 \mu \mathrm{m}$. (l) Hospitalization time in ISGlow patients versus ISGhigh patients. $(m)$ Representative IHC staining for complement activation products C5b-9 and C3d in ISGhigh, ISGlow COVID-19 and normal control lungs. Size bar $100 \mu \mathrm{m}$. ISGhigh samples, red; ISGlow samples, blue.

\section{Supplementary Files}

This is a list of supplementary files associated with this preprint. Click to download.

- 20200522Tables.docx

- Extendeddatafigure1v08.ai

- Extendeddatafigure2v06.ai

- Extendeddatafigure3v02.ai

- Extendeddatafigure4v03.ai

- Extendeddatafigure5v03.ai 